\title{
Frequency of Simvastatin Prescriptions With Potentially Interacting Medications in a Veterans Affairs Health Care System
}

\author{
JERILYN B. PETROPOULOS, BSPharm, PharmD, BCPS, and CRISTINA E. BELLO-QUINTERO, PharmD
}

\begin{abstract}
OBJECTIVE: The primary objective of this review is to quantify the proportion of patients on simvastatin, an HMG-CoA reductase inhibitor (commonly known as statin), who received concurrent prescriptions for potentially interacting chronicuse medications. The secondary objective is to determine the frequency with which simvastatin was prescribed above its recommended dose when administered concomitantly with known interacting medications.
\end{abstract}

METHODS: A retrospective review of computerized outpatient pharmacy records from a Veterans Affairs Medical Center and its associated ambulatory clinics was performed in September 2002.

RESULTS: A total of 12,240 patients had an active prescription for a statin. The majority of patients $(95 \%, N=11,677)$ were on simvastatin therapy, and 1,231 $(10.5 \%)$ of the patients on simvastatin were prescribed at least 1 potentially interacting medication. More than one half $(57.8 \%)$ of simvastatin doses were above the maximum recommended daily dose when prescribed with potentially interacting medications.

CONCLUSION: This analysis supports the need for vigilance in reviewing the dose of simvastatin in patients receiving interacting medications. Health care systems should consider strategies to educate health care professionals on prevention of drug interactions and adverse patient outcomes.

KEYWORDS: Simvastatin, Statins, Drug interactions, Myopathy, Drug use review

J Manag Care Pharm. 2004;10(3):239-243 $\square$ levated low-density lipoprotein (LDL) cholesterol has been identified as a primary risk-reduction target in patients at risk for coronary heart disease (CHD). Numerous epidemiological studies have demonstrated a relationship between elevated LDL and the incidence of CHD. Use of LDL-lowering therapy, including hydroxymethyl glutaryl coenzyme A (HMG-CoA) reductase inhibitors, also known as statins (atorvastatin, fluvastatin, lovastatin, pravastatin, rosuvastatin, and simvastatin), has been shown to significantly reduce risk for major coronary events and coronary deaths. Statins are recommended as standard treatment for elevated LDL and are widely used in clinical practice. ${ }^{1}$ Results from clinical trials with statins show a decrease in CHD and total mortality, revascularization procedures, and stroke. ${ }^{2-6} \mathrm{~A}$ meta-analysis demonstrates a decrease of $31 \%$ in major coronary events and $21 \%$ in total mortality with use of statins compared with placebo. ${ }^{7}$ Statins are generally well tolerated, with elevated liver enzymes and myopathy reported as the most common adverse effects. ${ }^{8.9}$

Myopathy is a general term for muscle disease and includes myalgia, myositis, and rhabdomyolysis (Figure 1). The prevalence of statin rhabdomyolysis is rare. A review of adverse drug events reported to the U.S. Food and Drug Administration (FDA) showed an overall reporting rate of 0.15 cases of fatal rhabdomyolysis per 1 million statin prescriptions. ${ }^{10}$ However, the true incidence of statin rhabdomyolysis is unknown because of the underreporting of adverse drug reactions during the postmarketing period of medications. All statins seem to have a potential for causing rhabdomyolysis. The risk of myopathy is dose related and increases with statin serum concentration. ${ }^{9,11}$ Most myopathy associated with statins occurs in patients of older age, small body frame, multisystem disease (including chronic renal insufficiency), multiple medications, perioperative periods, or specific drug-drug interactions. ${ }^{8,12,13}$

Several medications have been identified that increase the risk of myopathy when administered concurrently with statins, primarily by decreasing the metabolism of the statin (Figure 2). ${ }^{8,14-18}$ Although these drug-drug interactions are well documented as increasing risk for myopathy, published studies documenting the frequency of prescribing interacting drugs are lacking. Einarson and colleagues ${ }^{19}$ studied the extent of drug interactions and health care utilization in patients receiving statins. However, Einarson focused on concomitant drugs whose levels increased from the statin therapy and short-term concomitant therapy, which could increase statin serum concentrations.

The primary objective of this review is to quantify the proportion of patients in a primary care setting on simvastatin, the 


\section{FIGURE 1) Statin Myopathy}

\begin{tabular}{l|l}
\hline Myalgias & Muscle ache or weakness without CPK elevation* \\
\hline Myositis & Muscle symptoms with increased CPK levels \\
\hline Rhabdomyolysis & $\begin{array}{l}\text { Muscle symptoms with marked CPK elevation } \\
\text { (typically substantially greater than } 10 \text { times the upper } \\
\text { limit of normal) and with creatine elevation (usually } \\
\text { with brown urine and urinary myoglobin) }\end{array}$ \\
\hline
\end{tabular}

* Common reference ranges for creatine phosphokinase (CPK) are 40 to 200 international units/L for males and 35 to 150 international units/L for females. Local facility laboratory reference range is 55 to 170 international units/L.

\begin{tabular}{|c|}
\hline $\begin{array}{l}\text { FIGURE 2) Drugs With Potential } \\
\text { Interaction with Statins* }\end{array}$ \\
\hline Amiodarone \\
\hline Calcium channel blockers (diltiazem, verapamil) \\
\hline Cyclosporine \\
\hline Fibrates (gemfibrozil, fenofibrate) \\
\hline Niacin \\
\hline Potent inhibitors of the CYP3A4 enzyme system \\
\hline Azole antifungals (itraconazole, ketoconazole) \\
\hline Macrolide antibiotics (clarithromycin, erythromycin) \\
\hline $\begin{array}{l}\text { HIV protease inhibitors (amprenavir, atazanavir, fosamprenavir, } \\
\text { indinavir, nelfinavir, ritonavir, saquinavir) }\end{array}$ \\
\hline Nefazodone \\
\hline $\begin{array}{l}\text { *ases of rhabdomyolysis have also been reported with concomitant use with } \\
\text { digoxin or warfarin. However, it is not clear whether these cases were related } \\
\text { to statin-drug interactions. }\end{array}$ \\
\hline
\end{tabular}

\section{FIGURE 3 Recommended Maximum Doses of Simvastatin When Administered With Interacting Medications}

\begin{tabular}{l|c}
\hline Interacting Medication & $\begin{array}{l}\text { Maximum Recommended } \\
\text { Dose of Simvastatin (mg) }\end{array}$ \\
\hline Amiodarone & 20 \\
\hline Cyclosporine & 10 \\
\hline Fibrates (gemfibrozil or fenofibrate)* & 10 \\
\hline Niacin* & 10 \\
\hline Verapamil & 20 \\
\hline * Subsequent to preparation of this manuscript, the FDA approved a label change \\
for simvastatin to eliminate the maximum-dose warnings concerning concomi- \\
tant use with fenofibrate and niacin. The revised label still warns that the benefit \\
of further alterations in lipid levels by the combined use of simvastatin with \\
fibrates or niacin should be carefully weighed against the potential risks. \\
(U.S. Food and Drug Administration. Approval history for Zocor is available at \\
http://www.fda.gov/cder/ foi/appletter/2003/19766slr065,066ltr.pdf. Accessed \\
February 6, 2004.)
\end{tabular}

formulary statin at the time of this study, who had received concurrent prescriptions for potentially interacting chronic-use medications. The secondary objective is to determine the frequency with which simvastatin was prescribed above its recommended daily dose when administered concomitantly with known interacting medications.

\section{Methods}

This was a retrospective analysis of computerized outpatient records from a Veterans Affairs Medical Center and its associated ambulatory clinics, which had 429,500 outpatient visits in fiscal year 2002. Patients were included in the analysis if they had an active prescription for simvastatin at the time of review (September 2002). Pharmacy records, laboratory results, and patient demographics were retrieved for analysis from the computerized patient record.

The product labeling for simvastatin ${ }^{14}$ was reviewed to identify potentially interacting medications and dosage recommendations for concurrent use (Figure 3). At the time of this review, the manufacturer recommended a maximum daily dosing of $10 \mathrm{mg}$ for simvastatin when used concurrently with fibrates, niacin, or cyclosporine. A maximum daily simvastatin dose of $20 \mathrm{mg}$ is recommended when used concurrently with amiodarone or verapamil. The product labeling does not provide a maximum daily dose for simvastatin when administered concurrently with potent CYP3A4 (cytochrome P450, family 3, subfamily A, polypeptide 4) inhibitors. The manufacturer recommends generally avoiding the use of potent CYP3A4 inhibitors (Figure 2) unless the benefit of combined therapy outweighs the increased risk of myopathy and rhabdomyolysis.

At the time of this review, manufacturers did not provide specific dosage recommendations for atorvastatin and pravastatin, 2 other statins used in the study population, when prescribed concurrently with interacting medications. Although lovastatin did have new dosage recommendations for use with interacting drugs, simvastatin was the most frequently prescribed statin due to its formulary status at the time of this study. Therefore, atorvastatin, lovastatin, and pravastatin were excluded from the analysis.

Pharmacy records were queried for presence of concurrent prescriptions of potentially interacting medicines (Figure 2), including fibrates, niacin, cyclosporine, amiodarone, verapamil or chronic potent CYP3A4 enzyme inhibitors (nefazodone and protease inhibitors). Diltiazem was excluded from the analysis due to the absence of a maximum dose recommendation for concurrently prescribed simvastatin. Prescriptions for concomitant potent CYP3A4 enzyme inhibitors that are commonly used for acute situations (clarithromycin, erythromycin, itraconazole, and ketoconazole) were also not included in the analysis.

Statistical analysis was descriptive in nature and was performed in Microsoft Access. The Human Research Subcommittee determined this analysis to be exempt from Investigational Review Board review. 


\section{Results}

A total of 12,240 patients had an active prescription for a statin in the specified time period. Simvastatin was the most frequently prescribed statin (Table 1). Of the 11,677 patients receiving simvastatin, 1,231 (11\%) were prescribed at least 1 potentially interacting medication. Of these 1,231 patients, 1,176 (96\%) were prescribed 1 interacting medication, 54 patients (4\%) were prescribed 2 potentially interacting medications, and 1 patient was prescribed 3 potentially interacting medications. Overall, the majority $(57.8 \%)$ of simvastatin doses were above the maximum daily recommended dose when given with potentially interacting medications (Table 2 ).

A total of 75 simvastatin patients (mean dose $42 \pm 24 \mathrm{mg}$ ) also received nefazodone. Of these patients, 49 (65\%) received a dose greater than $10 \mathrm{mg}$ and $22(29 \%)$ received a dose greater than $20 \mathrm{mg}$. Two simvastatin patients also received protease inhibitors ( 1 patient was ordered nelfinavir, while the other was ordered both amprenavir and ritonavir); both of these patients were ordered a daily simvastatin dose of $40 \mathrm{mg}$. Pravastatin has less potential for interaction with potent inhibitors of CYP3A4 due to its lack of metabolism via the CYP450 pathway. It was prescribed for 14 patients who were also receiving protease inhibitors and 2 patients who were receiving nefazodone.

\section{Discussion}

This analysis supports the need for vigilance in reviewing the dose of simvastatin in patients receiving interacting medications. The dose-related nature of statin rhabdomyolysis is well established. Interactions with statins are also well documented in the literature as increasing the patient's risk for myopathy, primarily through decreasing the metabolism of statins and increasing their serum levels. The American College of Cardiology (ACC), American Heart Association (AHA), and National Heart, Lung, and Blood Institute (NHLBI) ${ }^{8}$ advise that "particular attention . . . be given to drug interactions" when using statin therapy. Merck submitted a supplemental new drug application to the FDA in May 2001, with subsequent labeling changes in response to postmarketing adverse event reports. ${ }^{20}$ Revisions to the simvastatin product label, effective May 2002, included additional warnings to describe the increased risk of rhabdomyolysis with use of specific concomitant drugs; maximum dose recommendations when simvastatin is used with cyclosporine, fibrates, niacin, amiodarone, and verapamil; recommendations to avoid concomitant use of simvastatin with potent CYP3A4 enzyme inhibitors; instructions to warn patients about the risk of myopathy; and a quantification of the dose-related effects of myopathy and rhabdomyolysis.

This study demonstrates a need to heighten awareness of dose-related simvastatin drug interactions. A significant number of simvastatin prescriptions were ordered at doses higher than the recommended maximum dose. The updated product labeling and ACC/AHA/NHLBI Clinical Advisory were pub-

\begin{tabular}{lr}
\hline TABLE 1) Patient Demographics \\
\hline \multicolumn{1}{c}{$\begin{array}{c}\text { Patients } \\
\mathbf{n}(\%)\end{array}$} \\
\hline Age & $70 \pm 11$ years \\
\hline Sex & \\
$\quad$ Male & $11,978(98)$ \\
Female & $262(2)$ \\
\hline Race & \\
White & $3,270(27)$ \\
Black & $787(6)$ \\
Hispanic & $513(4)$ \\
American Indian & $11(0.09)$ \\
Asian & $4(0.03)$ \\
Unknown & $7,655(63)$
\end{tabular}

\begin{tabular}{lr}
\hline $\begin{array}{l}\text { Most Recent CPK Result* } \\
\text { (reference range 55 U/L -170 U/L) }\end{array}$ \\
Not available & $10,460(85)$ \\
$<170$ U/L & $1,388(11)$ \\
Between 171U/L and 1,700 U/L & $389(3)$ \\
$>1,700$ U/L & $3(0.02)$ \\
\hline HMG-CoA Reductase Inhibitor ("Statin") & $283(2.3)$ \\
Atorvastatin & $233(1.9)$ \\
Lovastatin & $47(0.4)$ \\
Pravastatin & $11,677(95)$ \\
Simvastatin & mg \\
\hline Average Statin Dose & $42 \pm 29$ \\
Atorvastatin & $27 \pm 16$ \\
Lovastatin & $27 \pm 19$ \\
Pravastatin & $27 \pm 20$ \\
Simvastatin & \\
\hline * Most recent result from 9/1/01 to 9/1/02. \\
\hline
\end{tabular}

\section{TABLE 2 Simvastatin Dose With Concomitant Potentially Interacting Medications}

\begin{tabular}{|c|c|c|c|}
\hline $\begin{array}{l}\text { Patients } \\
\text { (n) }\end{array}$ & Concomitant Drug & $\begin{array}{l}\text { Dose of Simvastatin } \\
\text { Mean in } \mathrm{mg} \pm \mathrm{SD}\end{array}$ & $\begin{array}{l}\text { Patients Above } \\
\text { Maximum Daily } \\
\text { Dose }^{*} \text { n (\%) }\end{array}$ \\
\hline 509 & Gemfibrozil & $28 \pm 23$ & $362(71)$ \\
\hline 3 & Fenofibrate & $23 \pm 16$ & $2(66)$ \\
\hline 335 & Verapamil & $25 \pm 19$ & $92(27)$ \\
\hline 280 & Niacin & $32 \pm 24$ & $212(76)$ \\
\hline 98 & Amiodarone & $30 \pm 20$ & $39(40)$ \\
\hline 6 & Cyclosporine & $18 \pm 4$ & $5(83)$ \\
\hline 1,231 & & & $712(58)$ \\
\hline \multicolumn{4}{|c|}{$\begin{array}{l}\text { * Maximum dose recommended by the manufacturer in product labeling (May } \\
\text { 2002). }{ }^{6} \text { A total of } 57.8 \%(712 / 1,231) \text { of patients receiving concomitant potentially } \\
\text { interacting medications were prescribed simvastatin at a daily dose higher than } \\
\text { the maximum recommended. }\end{array}$} \\
\hline
\end{tabular}


lished very shortly before initiation of this review. Electronic interaction alerts currently display during the order-entry process to warn prescribers of these identified drug interactions with statins. As a result of this data, dose recommendations for simvastatin were added to the formulary information that is accessible when simvastatin is electronically ordered. A followup review is planned to compare it with the baseline data obtained from this analysis.

In this retrospective review, $11 \%$ of patients on simvastatin were prescribed at least 1 potentially interacting drug. Einarson and colleagues ${ }^{19}$ found a $15 \%$ prescribing rate for concomitant medications that can increase HMG-CoA reductase inhibitor concentrations and risk of myopathy. However, Einarson focused on acute therapy medications (erythromycin, clarithromycin, fluconazole, and ketoconazole) and antiulcer medications (cimetidine and omeprazole). Our review focused on chronic-use medications known to increase risk for HMG-CoA inhibitor (statin) myopathy. ${ }^{8,11-16}$ Patients prescribed short-term, potent CYP3A4 inhibitors were excluded from our review. Patients who receive short-term therapy with potent CYP3A4 inhibitors (e.g., clarithromycin, erythromycin, itraconazole, and ketoconazole) should be counseled to temporarily withhold their statin therapy.

In this analysis, $85 \%$ of patients did not have a creatine phosphokinase (CPK) level measured within the previous year. This is consistent with standard practice recommendations, as routine measurement of CPK levels is not required in asymptomatic patients receiving statins. ${ }^{8}$ Baseline CPK measurement is recommended to assist in the evaluation of subsequent myopathy. However, the date of initiation of statin therapy was not collected for analysis in this review.

Patients receiving statins should be asked at each follow-up visit if they are experiencing any unusual or unexpected muscle pain or tenderness. A follow-up CPK level should be obtained if the patient reports muscle symptoms, and it should be compared with baseline levels. Statin therapy should be discontinued if a patient with muscle pain has a CPK greater than 10 times the upper normal limit. If a patient experiences muscle pain with no CPK elevation or a moderate elevation in CPK, the patient's symptoms and CPK level should be followed weekly until there is no longer medical concern or symptoms worsen to the situation previously described. ${ }^{8}$ It should be noted that some statin patients do experience muscle weakness and histopathologic findings of myopathy despite a normal CPK. ${ }^{21}$

As with any medical therapy, the risks and benefits of statin use must be carefully considered. In patients with hyperlipidemia, statin therapy has demonstrated an approximate onethird decrease in coronary deaths and major coronary events compared with placebo in clinical trials. Conversely, the absolute risk of rhabdomyolysis is low, with fewer than 0.15 cases of fatal rhabdomyolysis reported per 1 million statin prescriptions. Careful patient selection, counseling, and monitor- ing can reduce the risk of statin myopathy while allowing patients to receive the positive benefits of statin therapy. High-dose statin therapy should be avoided in patients at increased risk of myopathy, including those on potentially interacting medications. In patients who are prescribed medications that inhibit the CYP3A4 enzyme system, using statins that are not metabolized by the CYP3A4 enzyme system (fluvastatin, pravastatin, and rosuvastatin) may minimize risk of rhabdomyolysis.

\section{Limitations}

The primary objective of this analysis was to quantify the proportion of patients on simvastatin who had received concurrent prescriptions for potentially interacting medications. Although the existence of potential drug interactions was identified, patient progress notes were not reviewed for outcomes of potential myopathy. Review of reported adverse drug reactions revealed 25 patients with simvastatin myopathy during fiscal year 2001 and fiscal year 2002 at our institution; these include 3 reports of rhabdomyolysis, 4 of myositis, and 18 of myalgias. Twenty percent of these patients ( 5 of 25) were also receiving interacting medications. High-dose simvastatin with an interacting medication, $80 \mathrm{mg}$ daily with concurrent gemfibrozil, was implicated in 1 of the reports of rhabdomyolysis. The other reports with interacting medications were all cases of myalgia. These adverse drug reactions are voluntarily reported and may underestimate the actual numbers of patients with statin myopathy. Further research should be conducted to quantify the outcome of statin drug interactions in the managed care setting.

This analysis is limited by its retrospective design and method of data collection. Information was retrieved from a computer search of prescription, laboratory, and demographic records. Patient race was not identified in 63\% of cases; therefore, no ethnicity analysis can be performed. Patient progress notes were not reviewed to determine the extent of patient counseling that was provided. Medication adherence was also not determined.

\section{Conclusion}

The risk of HMG-CoA reductase inhibitor (statin) myopathy is often associated with concomitant use of other medications. This retrospective analysis in a population of elderly males demonstrates a high frequency of coadministration of simvastatin with potentially interacting drugs, often above the recommended maximum dose of simvastatin. Health care systems need to initiate strategies to educate health care professionals on minimizing or preventing drug interactions, in an effort to reduce the risk of negative patient outcomes.

\section{ACKNOWLEDGMENTS}

The authors gratefully acknowledge Richard Spekis, computer programmer, Department of Veterans Affairs Medical Center, Miami, Florida, for his expertise in information technology. 


\section{DISCLOSURES}

No outside funding supported this research. Author Jerilyn B. Petropoulos served as principal author of the study. Study concept and design and statistical expertise were contributed by Petropoulos. Analysis and interpretation of data and drafting of the manuscript were contributed by Petropolous and author Cristina E. Bello-Quintero; critical revision of the manuscript was the work of Bello-Quintero.

\section{REFERENCES}

1. Executive Summary of the Third Report of the National Cholesterol Education Program (NCEP) Expert Panel on Detection, Evaluation, and Treatment of High Blood Cholesterol in Adults (Adult Treatment Panel III). JAMA. 2001;285(19):2486-97.

2. Downs JR, Clearfield M, Weis S, et al. Primary prevention of acute coronary events with lovastatin in men and women with average cholesterol levels: results of AFCAPS/TexCAPS. JAMA. 1998;279:1615-22.

3. Scandinavian Simvastatin Survival Study Group. Randomised trial of cholesterol lowering in 4,444 patients with coronary heart disease: the Scandinavian Simvastatin Survival Study (4S). Lancet. 1994;344:1383-89.

4. Shepherd J, Cobbe SM, Ford I, et al., for the West of Scotland Coronary Prevention Study Group. Prevention of coronary heart disease with pravastatin in men with hypercholesterolemia. N Engl J Med. 1995;333:1301-07.

5. Sacks FM, Pfeffer MA, Moye LA, et al., for the CARE investigators. The effect of pravastatin on coronary events after myocardial infarction in patients with average cholesterol levels. N Engl J Med. 1996;335:1001-09.

6. Long-Term Intervention with Pravastatin in Ischaemic Disease (LIPID) Study Group. Prevention of cardiovascular events and death with pravastatin in patients with coronary heart disease and a broad range of initial cholesterol levels. N Engl J Med. 1998;339:1349-57.

7. LaRosa JC, He J, Vuppurturi S. Effect of statins on risk of coronary disease: a meta-analysis of randomized controlled trials. JAMA. 1999;282:2340-46.

8. Pasternak RC, Grundy SM, Smith SC, et al. ACC/AHA/NHLBI Clinical advisory on the use and safety of statins. J Am Coll Cardiol. 2002;40(3):567-72.
9. Smith CC, Bernstein LI, Davis RB, et al. Screening for statin-related toxicity: the yield of transaminase and creatine kinase measurements in a primary care setting. Arch Intern Med. 2003;163:688-92.

10. Staffa JA, Chang J, Green L. Cerivastatin and reports of fatal rhabdomyolysis. N Engl J Med. 2002;346:539-40.

11. Omar MA, Wilson JP, Cox TS. Rhabdomyolysis and HMG-CoA reductase inhibitors. Ann Pharmacother. 2001;35:1096-1107.

12. Thompson PD, Clarkson P, Karas RH. Statin-associated myopathy. JAMA. 2003;289:1681-90.

13. Ballantyne CM, Corsini A, Davidson MH, et al. Risk for myopathy with statin therapy in high-risk patients. Arch Intern Med. 2003;163:553-64.

14. Zocor [package insert]. Whitehouse Station, NJ: Merck \& Co, Inc; May 2002.

15. Omar MA, Wilson JP. FDA adverse event reports on statin-associated rhabdomyolysis. Ann Pharmacother. 2002;36:288-95.

16. Black C, Jick H. Etiology and frequency of rhabdomyolysis. Pharmacotherapy. 2002;22(12):1524-26.

17. Gruer PJK, Vega JM, Mercuri MF, et al. Concomitant use of cytochrome P450 3A4 inhibitors and simvastatin. Am J Cardiol. 1999;84:811-15.

18. Bottorff M, Hansten P. Long-term safety of hepatic hydroxymethyl glutaryl coenzyme A reductase inhibitors: the role of metabolism-monograph for physicians. Arch Intern Med. 2000;160(15):2273-80.

19. Einarson TR, Metge CJ, Iskedjian M, et al. An examination of the effect of cytochrome P450 drug interactions of hydroxymethylglutaryl-coenzyme A reductase inhibitors on health care utilization: a Canadian population-based study. Clin Ther. 2002;24(12):2126-36.

20. Food and Drug Administration. Approval history for Zocor at drugs@fda. Available at: http://www.accessdata.fda.gov/scripts/cder/drugsatfda/index.cfm? fuseaction=Search.ApprovalHistoryFDA.gov. Accessed February 6, 2004.

21. Phillips PS, Haas RH, Bannykh S, et al. Statin-associated myopathy with normal creatine kinase levels. Ann Intern Med. 2002;137:581-85. 